\title{
MONISEDA project. Improving analgosedation monitoring in Spanish pediatric intensive care units.
}

\section{Santiago Mencia ( $\nabla$ santiagomencia@gmail.com )}

Gregorio Marañón General University Hospital https://orcid.org/0000-0001-7786-688X

\section{Raquel Cieza}

General University hospital Gregorio Maranon: Hospital General Universitario Gregorio Maranon

Jimena Del Castillo

Hospital General Universitario Gregorio Marañón: Hospital General Universitario Gregorio Maranon

Jesús López-Herce

Hospital General Universitario Gregorio Maranon

Sedation Group of Spanish Pediatric Critical Care SEDUCIP

General University hospital Gregorio Maranon: Hospital General Universitario Gregorio Maranon

\section{Research}

Keywords: monitoring, analgosedation, MONISEDA project, PICU

Posted Date: July 13th, 2021

DOI: https://doi.org/10.21203/rs.3.rs-666544/v1

License: (c) (1) This work is licensed under a Creative Commons Attribution 4.0 International License. Read Full License 


\section{Abstract \\ Background}

Analgosedation (AS) assessment using clinical scales is crucial to follow the international recommendations about analgosedation. The Analgosedation workgroup of the Spanish Society of Pediatric Intensive Care (SECIP) carried out two surveys in 2008 and 2015, which verified the gap in analgosedation assessment in Spanish PICUs. The objective of the study was to analyze how analgosedation assessment by clinical scales changed after a multicenter intervention program.

\section{Methods}

multicenter pre-post study comparing the use of sedation, analgesia, withdrawal and delirium scales before and after the MONISEDA project. Results were also compared with a control group formed by nonparticipating units. A survey about analgosedation management and monitoring was filled out before (year 2015) and after (year 2020) the implementation of the Moniseda project in 2016. Results were compared between those periods of time, but also between participant and non-participants PICUs in the Moniseda project (M-group and non-M group, respectively). Data related to analgosedation of all patients admitted to a Moniseda-participant PICU were also collected for 2 months.

\section{Results}

15 Spanish PICUs were enrolled in the Moniseda project and other 15 non-participant PICUs formed the control group. In the M-group, the number of PICUs with a written analgosedation protocol increased from 53 to $100 \%(p=0.003)$ and withdrawal protocol from 53 to $100 \%(p=0.003)$, whereas in non-M group the written AS protocol increased from 80 to $87 \%$ and withdrawal protocol stayed on $80 \%$.

\section{Key Points Summary}

Question: Could a national project improve the analgosedation assessment in the Spanish PICU?

Findings: A multicenter training projects called MONISEDA project, could be an effective tool to achieve a better analgosedation assessment of critically ill children.

Meaning: Our findings suggest that MONISEDA project could serve as a model for other countries, adjusting it their specific characteristics.

\section{Glossary Of Terms}

AS: analgosedation; SECIP: Spanish Society of Pediatric Intensive Care; IWS: iatrogenic withdrawal syndrome; BIS: biespectral index. PICU: pediatric intensive care unit. 


\section{Introduction}

Sedation and analgesia are essential in the management of critically ill children. Pain and anxiety abolition in children must be a priority. An adequate analgosedation diminishes emotional stress, facilitates nursing care, adaptation to mechanical ventilation and improves prognosis, reducing the length of mechanical ventilation and PICU stay $(1,2)$. However, sedative and analgesic drugs can cause adverse effects and increase morbidity and mortality (3).

International recommendations highlight the importance of improving comfortability in critically ill children, mainly through proper analgesia, minimal possible sedation, and measures to prevent withdrawal syndrome and delirium $(4,5)$. For that purpose, it is necessary to assess and treat pain prior to administration of sedatives, and to keep minimal sedation to allow patients to interact with the environment without agitation. It is fundamental to apply valid and reliable assessment tools to identify pain, excessive or insufficient sedation and delirium in critically ill children, and to use them on a routine basis, adjusting our procedures according to its rating $(6,7,8,9)$.

The Analgosedation workgroup of the Spanish Society of Pediatric Intensive Care (SECIP) carried out two surveys in 2008 and 2015, which verified the gap in analgosedation assessment in Spanish PICUs. Therefore, the group decided to perform a training program called MONISEDA project. Its objectives were to create analgosedation working teams in each Spanish PICU and to promote and unify analgosedation clinical scales to improve the assessment of pain, stress, iatrogenic withdrawal syndrome and delirium.

\section{Methods}

A multicenter pre-post study comparing the use of sedation, analgesia, withdrawal and delirium scales before and after the MONISEDA project was performed. The project was advertised on the SECIP website and all the Spanish PICUs that were interested had the opportunity to participate in it. The Institutional Review Board reviewed the study and approved it (EPA-SP 02/2017) and written informed consent was waived. This manuscript adheres to the applicable STROBE guidelines.

The Moniseda project was divided into different stages:

1. Preliminary survey. A survey was performed in 2015 to determine how pain and stress was being managed and monitored in PICUs at that moment. Two groups were included: PICUs participating in Moniseda project (M-group) and non-participants (non-M group).

2. Development of MONISEDA training program (Table 1): including informative and training activities with sessions and workshops for all PICU members for a period of 2 months. The project encouraged the creation of an analgosedation team in each PICU of the M-group, consisting of 1 or 2 doctors and 4-6 nurses. 
Table 1

Phases of the MONISEDA project training in each PICU.

\begin{tabular}{|c|c|c|}
\hline Periods & Duration & Description \\
\hline \multirow[t]{2}{*}{$\begin{array}{l}\text { Analgosedation } \\
\text { teams }\end{array}$} & 15 days & $\begin{array}{l}\text { - Creation of a team in each PICU that will be composed of } 1 \text { or } 2 \\
\text { physicians and } 4 \text { to } 6 \text { PICU nurses. }\end{array}$ \\
\hline & & $\begin{array}{l}\text { - Implementation or reinforcement of scales and analgosedation } \\
\text { protocol. }\end{array}$ \\
\hline $\begin{array}{l}\text { Information } \\
\text { period }\end{array}$ & 15 days & $\begin{array}{l}\text { - Presentation of the project to the rest of the PICU staff members via: } \\
\text { - General session. } \\
\text { - Workshops regarding clinical scales of analgesia, sedation, IWS } \\
\text { and delirium (driven by Analgosedation team nurses). }\end{array}$ \\
\hline \multirow[t]{2}{*}{ Training period } & 1 month & $\begin{array}{l}\text { - All nurses will assess and register analgesia, sedation, iatrogenic } \\
\text { withdrawal syndrome and delirium using scales once per shift. }\end{array}$ \\
\hline & & -All doubts will be discussed with the Analgosedation team members. \\
\hline \multirow[t]{2}{*}{$\begin{array}{l}\text { Data collection } \\
\text { period }\end{array}$} & $\begin{array}{l}2 \\
\text { months }\end{array}$ & $\begin{array}{l}\text { - Data collection from each patient concerning analgosedation by } \\
\text { specific team members. }\end{array}$ \\
\hline & & - Data analysis and evaluation of results. \\
\hline
\end{tabular}

The analgosedation working team was responsible for the training of the rest of the PICU staff through an informative clinical session, and a practical training workshop of the use of clinical scales and data collection. Specially addressed to the nurses of the unit.

3. Data collection phase: for 2 months, PICUs participating in Moniseda project filled in the data collection form. After obtaining informed consent from parents or guardians, the scores for the analgesia clinical scale (adapted to age) and sedation (COMFORT scale) for all children admitted to the PICU were registered once per shift $(6 \mathrm{am}, 2 \mathrm{pm}, 9 \mathrm{pm})$. No patients were excluded. Data was sent to the coordinator center for its analysis. All study coordinators from the different PICUs were asked to complete a satisfaction survey upon completion of this phase.

4. Subsequent survey after the project. In 2020 , the same data collection form was again completed by all the PICUS of both groups, in order to compare these results with the previous ones.

\section{Statistical analysis}

All data was analyzed by the statistical package SPSS for Windows, version 19. Qualitative variables were expressed as percentages, and quantitative variables, as means and standard deviation. Fisher exact test was used to compare qualitative variables, and Mann-Whitney $U$ test for quantitative variables. The McNemar test for related samples was used to analyze the evolution of the variables of the 2020 survey with respect to that of 2015 . Statistical signification was considered when $p<0,05$. 


\section{Results}

\section{Analgosedation survey in 2015:}

Table 2 shows the results of the first sedoanalegia survey, comparing PICUs of the M-group and the non$\mathrm{M}$ group. The number of PICUs that followed a written analgosedation and withdrawal protocol was higher in the non-M group, although differences were not statistically significant.

Table 2

Initial analgosedation survey (year 2015). Comparison between MONISEDA group and nonMONISEDA group.

\begin{tabular}{|lllll|}
\hline Variable & $\begin{array}{l}\text { Global results } \\
(\%)\end{array}$ & Moniseda (\%) & Non-Moniseda (\%) & P \\
\hline Written AS protocol & 67 & 53 & 80 & 0.123 \\
\hline Use of daily AS scales & 10 & 7 & 13 & 0.5 \\
\hline AS working team creation & 10 & 7 & 13 & 0.5 \\
\hline Sedation scale used & 71 & Ramsay & Ramsay & 0.5 \\
\hline Objective monitoring: BIS & 30 & 76 & 66 & 0.5 \\
\hline Written WS protocol & 67 & 33 & 27 & 0.123 \\
\hline Use of daily IWS scales & 3 & 53 & 80 & 0.5 \\
\hline Usual use of delirium scales & 0 & 7 & 0 & - \\
\hline AS: analgosedation; BIS: biespectral index; IWS: iatrogenic withdrawal syndrome & \\
\hline
\end{tabular}

\section{MONISEDA Project 2016:}

In the M-group, the project was introduced to the rest of the staff in $85 \%$ of the PICUs, and a specific analgosedation working team consisting of doctors and nurses was created in $61 \%$ of the units.

At the end of the project, a satisfaction survey was completed by the coordinators of each PICU (Table 3 ). Main difficulties to implement monitoring were the lack of habit and the workload, principally from the nurses' point of view (40\%). $33 \%$ of doctors and $31 \%$ of nurses thought that the project had significantly changed routine analgosedation monitoring, and $70 \%$ of participants considered that some monitoring aspects had changed. The most important improvement was the incorporation of the use of clinical monitoring scales in $33 \%$ of the units. A greater use of sedation scales was attained in ten PICUs, of withdrawal scales in six units and of analgesia scales in three units. Four PICUs started to use delirium scales. 
Table 3

Satisfaction survey of MONISEDA group 2016.

\begin{tabular}{|ll|}
\hline Variable & MONISEDA Project (\%) \\
\hline AS working team creation & 61.5 \\
\hline Difficulties to develop the project & 40 \\
\hline Changes in AS daily management & 69 \\
\hline Daily AS monitoring implementation & 33 \\
\hline Daily IWS monitoring implementation & 40 \\
\hline AS: analgosedation; IWS: iatrogenic withdrawal syndrome \\
\hline
\end{tabular}

Table 4. Final analgosedation survey (year 2020). Comparison between MONISEDA group and nonMONISEDA group.

\begin{tabular}{|lllll|}
\hline Variable & $\begin{array}{l}\text { Global results } \\
(\%)\end{array}$ & $\begin{array}{l}\text { Moniseda } \\
(\%)\end{array}$ & Non-Moniseda (\%) & P \\
\hline Written AS protocol & 93 & 100 & 87 & 0.5 \\
\hline Use of daily AS scales & 100 & 100 & 100 & - \\
\hline AS working team creation & 40 & 47 & 33 & 0.355 \\
\hline Sedation scale used & & COMFORT & COMFORT & \\
\hline Objective monitoring: BIS & 60 & 100 & 66 & 0.645 \\
\hline Written IWS protocol & 90 & 60 & 60 & 0.241 \\
\hline Use of daily WS scales & 57 & 100 & 80 & 0.001 \\
\hline Usual use of delirium scales & 20 & 87 & 27 & 0.080 \\
\hline
\end{tabular}

AS: analgosedation; BIS: biespectral index; IWS: iatrogenic withdrawal syndrome

During the 2 months of the study, data from 489 children were collected. $55 \%$ were males. Mean age was 4.2 years old (SD 4.7) and mean weight $21 \mathrm{Kg}$ (SD 18). The reason for admission was medical pathologies in $53 \%$ of the cases. Mean length of stay was 6,3 days (SD 13). $30 \%$ of the patients underwent mechanical ventilation and $1.8 \%$ of the patients died.

Analgesia was monitored by scales in $97 \%$ of the patients, with a mean score of 1.5 (SD 1.4). Sedation assessment was performed by COMFORT scale in $93 \%$ of the patients, with an average rating of 18.3 (SD 
5.9). Bispectral index (BIS) monitoring of the level of consciousness was used in $8 \%$ of the patients, with a mean score of 56 (SD 14).

\section{Analgosedation survey in 2020:}

Table 4 shows the results of the last analgosedation survey performed, comparing PICUs of the M-group and the non-M group. Every PICU (100\%) in both groups used some analgosedation clinical scale on a daily basis. PICUs in the M-group performed analgosedation assessment more frequently than the control group. Statistically significant differences were found for withdrawal monitoring ( $87 \%$ vs $27 \% ; p=0.001$ ). Delirium assessment increased importantly too $(33 \%$ vs $7 \% ; p=0.08)$ but did not reach statistical significance.

\section{Comparison between 2015 and 2020 surveys.}

Figure 1 shows the comparison between the first and the last analgosedation survey. In both groups the use of clinical scales improved. The increase of analgosedation monitoring activities was higher in the Mgroup than in the non-M group. There was a higher increment in the creation of analgosedation working teams and written protocols for withdrawal monitoring.

\section{Discussion}

This is the first multicenter project that aims to improve analgosedation monitoring in Spanish PICUs. Our study shows that a specific training project significantly improves analgesia, sedation and withdrawal monitoring. It enhances awareness of health professionals and facilitates the creation of analgosedation working teams consisting of doctors and nurses. This leads to regular and long-term monitoring after the educational intervention. Our project could be a model for the development of new similar projects in other countries.

In the second survey, conducted five years after the intervention, it is important to highlight the improvement in the daily monitoring of analgosedation, withdrawal and delirium. Creation of multidisciplinary working teams (doctors and nurses), and a better follow-up of the recommendations to homogenize the use of clinical scales in the Spanish PICUs had significantly increased too. Furthermore, the use of COMFORT/COMFORT-b scale (specific for pediatric patients) raised compared to Ramsay scale, which is only validated for adults $(10,11)$.

The analysis showed that over the 2 months of data collection, analgosedation monitoring followed the international recommendations. The implementation of analgesia scales per shift achieved good pain control. Based on an early diagnosis and treatment adjustment according to the score, most of the patients showed no pain or mild pain, and an appropriate level of sedation (4).

The second survey, conducted five years after the intervention, showed an improvement of analgosedation monitoring in both the $\mathrm{M}$ and non-M group, which reflected a growing awareness of PICU health professionals of the importance of this monitoring $(12,13)$. We think that there could have been a 
contagion or spread effect from the PICUs included in the MONISEDA project to the rest of Spanish PICUs $(14,15)$.

Other studies have previously highlighted the importance of an appropriate AS monitoring in order to prevent and manage the appearance of withdrawal syndrome or delirium (16-18).

Achieving an improvement on AS and withdrawal monitoring in the Spanish PICUs is challenging. The implementation of a new routine in a clinical service is difficult, especially when it is a highly complex unit and there is a high staff turnover. Both time and a great effort are essential to accomplish this task. Difficulties to introduce these types of protocols are mentioned in other studies $(19,20)$. We consider that the creation of working teams made up of doctors and nurses is very important. The engagement and training of the nursing staff is crucial as they are in charge of the AS monitoring and the adjustment of the treatment to the patient's condition (16). However, despite the observed improvement in 2020 survey, there are still some aspects, as the withdrawal and delirium monitoring $(16,21,22)$ which need to be enhanced and that require continuous evaluation and feedback.

Our study has some limitations. Despite that every Spanish PICU was invited to participate in the project, only one third of them accepted. So, probably, those PICUs included in the project were also those with higher awareness of the importance of analgosedation and those that felt the need to implement these

protocols. This concern could have introduced a bias in the comparison of both groups and could explain the fact that in the initial survey only a small percentage of PICUs followed a written AS protocol. Another limitation is that the observational study of patients was not repeated in 2020 to verify the improvements observed in the survey in the daily practice.

\section{Conclusions}

In conclusion, we think that the creation of multicenter training projects, like MONISEDA project, could be an effective tool to achieve a better analgosedation assessment of critically ill children. Our project could serve as a model for other countries, adjusting it their specific characteristics.

\section{Declarations}

Competing interests: All authors have disclosed that they do not have any potential conflicts of interest.

\section{Authors' contributions:}

Santiago Mencía: This author helped in manuscript writing, study design, programming, data acquisition, and data validation.

Raquel Cieza: This author helped as methodology advisor and with manuscript editing.

Jimena del Castillo: This author helped with manuscript editing. 
Jesús López-Herce: This author helped in study design and in manuscript writing.

Sedation Group of Spanish Pediatric Critical Care Society (SECIP)*: These authors helped in data acquisition, and data validation.

Ethical Approval and Consent to participate: The Institutional Review Board reviewed the study and approved it (EPA-SP 02/2017).

Word count in text: 1,723

Date sent: 01/02/2021

Written informed consent was waived. This manuscript adheres to the applicable STROBE guidelines

Funding: No external funding was obtained for this study.

Consent for publication: We consent to the publication of the manuscript.

Availability of supporting data: All the data from the study are available.

Acknowledgements: Sedation Group of Spanish Pediatric Critical Care Society (SECIP).

\section{References}

1. Payen J-F, Bosson J-L, Chanques G, Mantz J, Labarere J, Investigators DOLOREA. Pain assessment is associated with decreased duration of mechanical ventilation in the intensive care unit: a post Hoc analysis of the DOLOREA study. Anesthesiology. 2009;111:1308-16.

2. Chanques $G$, Jaber $S$, Barbotte $E$, et al. Impact of systematic evaluation of pain and agitation in an intensive care unit. Crit Care Med. 2006;34:1691-9.

3. Baarslag MA, Jhingoer S, Ista E, Allegaert K, Tibboel D, van Dijk M. How often do we perform painful and stressful procedures in the paediatric intensive care unit? A prospective observational study. Aust Crit Care. 2019;32:4-10.

4. Harris J, Ramelet A-S, van Dijk M, et al. Clinical recommendations for pain, sedation, withdrawal and delirium assessment in critically ill infants and children: an ESPNIC position statement for healthcare professionals. Intensive Care Med. 2016;42:972-86.

5. Vet NJ, Ista E, de Wildt SN, van Dijk M, Tibboel D, de Hoog M. Optimal sedation in pediatric intensive care patients: a systematic review. Intensive Care Med. 2013;39:1524-34.

6. Dorfman TL, Sumamo Schellenberg E, Rempel GR, Scott SD, Hartling L. An evaluation of instruments for scoring physiological and behavioral cues of pain, non-pain related distress, and adequacy of analgesia and sedation in pediatric mechanically ventilated patients: A systematic review. Int J Nurs Stud. 2014;51:654-7. 
7. Van Dijk M, de Boer JB, Koot HM, et al. The association between physiological and behavioral pain measures in 0- to 3-year-old infants after major surgery. J Pain Symptom Manage. 2001;22:600-9.

8. Carnevale FA, Razack S. An item analysis of the COMFORT scale in a pediatric intensive care unit. Pediatr Crit Care Med. 2002;3:177-80.

9. Spagrud LJ, Piira T, Von Baeyer CL. Children's self-report of pain intensity. Am J Nurs. 2003;103:624.

10. Maaskant J, Raymakers-Janssen P, Veldhoen E, Ista E, Lucas C, Vermeulen H. The clinimetric properties of the COMFORT scale: A systematic review. Eur J Pain. 2016;20:1587-611.

11. Boerlage AA, Ista E, Duivenvoorden HJ, de Wildt SN, Tibboel D, van Dijk M. The COMFORT behaviour scale detects clinically meaningful effects of analgesic and sedative treatment. Eur J Pain. 2015;19:473-9.

12. Saliski M, Kudchadkar SR. Optimizing Sedation Management to Promote Early Mobilization for Critically III Children. J Pediatr Intensive Care. 2015;4:188-93.

13. Vincent JL, Shehabi Y, Walsh TS, et al. Comfort and patient-centred care without excessive sedation: the eCASH concept. Intensive Care Med. 2016;42:962-71.

14. Mencía S, García-San Prudencio M, Tapia R, et al. Monitoring and management of sedoanalgesia in Spanish pediatric intensive care units. MONISEDA proyect. Abstract P-487. 9th Congress of the World Federation of Pediatric Intensive \& Critical Care Societies Singapore, 10 de junio 2018. Pediatric Critical Care Medicine. 19 (6S):200.

15. Mencía S, García M, Tapia R. and grupo SEDUCIP de la SECIP. "Proyecto MONISEDA. Monitorización y manejo de la sedoanalgesia en las UCIP españolas". XXXIII Congreso de la Sociedad de Cuidados Intensivos Pediátricos. Granada, 10-13 junio 2018.

16. Neunhoeffer F, Kumpf M, Renk $\mathrm{H}$, et al. Nurse-driven pediatric analgesia and sedation protocol reduces withdrawal symptoms in critically ill medical pediatric patients. Paediatr Anaesth. 2015:786-94.

17. Kudchadkar SR, Yaster M, Punjabi NM. Sedation, sleep promotion, and delirium screening practices in the care of mechanically ventilated children: a wake-up call for the pediatric critical care community. Crit Care Med. 2014;42:1592-600.

18. Sanavia E, Mencía S, Lafever SN, Solana MJ, Garcia M, López-Herce J. Sedative and Analgesic Drug Rotation Protocol in Critically III Children with Prolonged Sedation: Evaluation of Implementation and Efficacy to Reduce Withdrawal Syndrome. Pediatr Crit Care Med. 2019;20:1111-17.

19. Franck LS, Naughton I, Winter I. Opioid and benzodiazepine withdrawal symptoms in paediatric intensive care patients. Intensive Crit care Nurs. 2004;20:344-51.

20. Keogh SJ, Long DA, Horn DV. Practice guidelines for sedation and analgesia management of critically ill children: a pilot study evaluating guideline impact and feasibility in the PICU. BMJ Open. 2015;30(3):e006428. 5 ).

21. Deeter KH, King MA, Ridling D, Irby GL, Lynn AM, Zimmerman JJ. Successful implementation of a pediatric sedation protocol for mechanically ventilated patients. Crit Care Med. 2011;39:683-8. 
22. Curley MAQ, Gedeit RG, Dodson BL, et al; RESTORE Investigative Team. Methods in the design and implementation of the Randomized Evaluation of Sedation Titration for Respiratory Failure (RESTORE) clinical trial [published correction appears in Trials. 2019; 7; 20: 17.

\section{Figures}

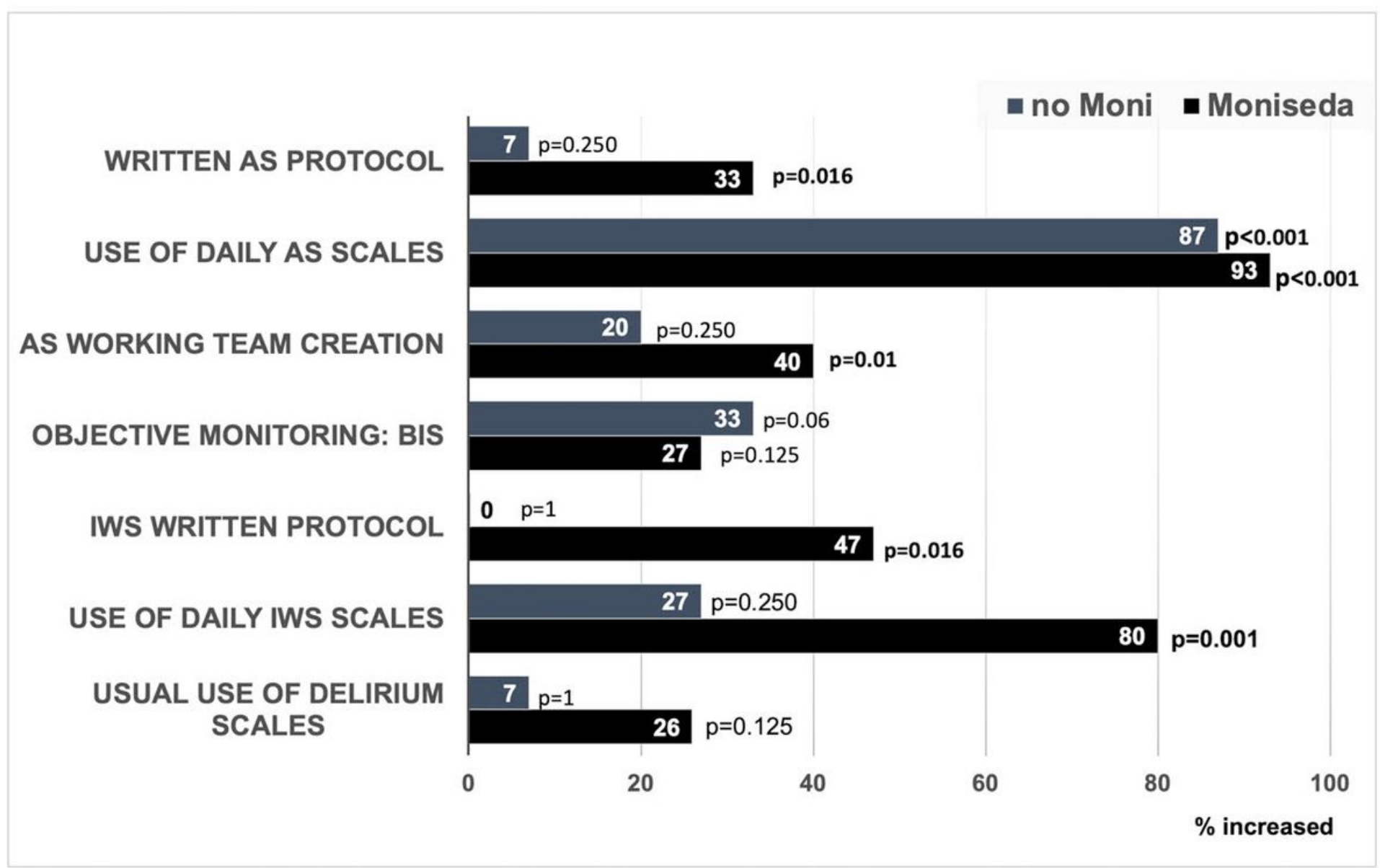

AS: analgosedation; BIS: biespectral index; IWS: iatrogenic withdrawal syndrome

\section{Figure 1}

Improvement in analgosedation survey 2020 compared to 2015 in both MONISEDA and non-MONISEDA groups. 